\title{
Synchronization phase as an indicator of persistent quantum correlations between subsystems
}

\author{
Stefan Siwiak-Jaszek $\odot,{ }^{*}$ Thao P. Le $\odot$, and Alexandra Olaya-Castro $\oplus^{\dagger}$ \\ Department of Physics and Astronomy, University College London, London WC1E 6BT, United Kingdom
}

(Received 8 July 2020; accepted 31 August 2020; published 22 September 2020)

\begin{abstract}
Spontaneous synchronization is a collective phenomenon that can occur in both dynamical classical and quantum systems. Here, we analyze the spontaneous synchronization dynamics of vibrations assisting energy transfer in a bio-inspired system. We find the emergence of a constant nonzero "synchronization phase" between synchronized vibrational displacements as the natural frequencies of the oscillators are detuned. This phase difference arises from the asymmetric participation of local modes in the long-lived synchronized state. Furthermore, we investigate the relationships between the synchronization phase, detuning and the degree of quantum correlations between the synchronizing subsystems and find that the synchronization phase captures how quantum correlations persistently exceed classical correlations during the dynamics. We show that our analysis applies to a variety of spontaneously synchronizing open quantum systems. Our work therefore opens up a promising avenue to investigate nontrivial quantum phenomena in complex biomolecular and nanoscale chemical systems.
\end{abstract}

DOI: 10.1103/PhysRevA.102.032414

\section{INTRODUCTION}

When two oscillators interact, they can undergo synchronization, where their frequencies align [1], usually with a nonzero phase difference. Synchronization is a well-studied classical phenomenon that appears recurrently throughout the natural world [2,3]. More recently it has been explored for physical systems in the quantum domain [4-11]. Oscillating quantum systems with a stable limit cycle can synchronize in the steady state to an external driving field [8] or to another quantum system [9]. The latter is often referred to as spontaneous synchronization [12]. In the absence of any external drive, quantum systems can also undergo transient spontaneous quantum synchronization during the early-time dynamics prior to relaxation to the ground state [12-17]. The underlying mechanisms leading to this transient behavior are effective collective dissipation processes [14] or combined local dissipation and system-system interactions $[12,16]$. Transient spontaneous quantum synchronization is particularly interesting in bio-inspired quantum settings because it allows us to understand the possible roles of quantum coherence in such systems [18]. Furthermore, transient spontaneous synchronization has been linked to the appearance of quantum correlations $[12,14,16]$, suggesting they may be necessary for synchronization to occur [12]. However, research on transient spontaneous quantum synchronization rarely focuses on the nature of phase itself between synchronized systems, despite its classical relevance.

Classically, the "robustness" of synchronization refers to the ability of oscillators to lock in phase despite their different natural frequencies: within a certain range of detuning $\Delta v$, the

\footnotetext{
*stefan.siwiak-jaszek.11@ucl.ac.uk

†a.olaya@ucl.ac.uk
}

oscillators are able to lock into a new resultant frequency that lies between the original frequencies. The equation of motion for the phase difference between two weakly coupled classical oscillators to first order is [1]

$$
\frac{d \Delta \phi(t)}{d t}=-\Delta v+\epsilon f(\Delta \phi(t))
$$

where $\Delta \phi(t)$ is the phase difference between oscillations, $\epsilon$ is a constant proportional to their coupling strength, and $f(\cdot)$ is a time-dependent periodic function. Synchronization can only occur if the detuning lies between the min and max extrema of $f(\Delta \phi(t))$ :

$$
\min _{\phi} \epsilon f(\Delta \phi(t))<\Delta v<\max _{\phi} \epsilon f(\Delta \phi(t)) .
$$

Within this detuning region, there is at least one stationary solution to Eq. (1) corresponding to synchronization. In most cases this synchronization occurs with a nonzero phase. We refer to this phase difference as the synchronization phase. As the detuning $\Delta v$ increases, the synchronization degrades and eventually disappears.

Quantum-mechanical oscillators typically exhibit analogous behavior regarding detuning and synchronization to their classical counterparts as described above [14]. Analytic equations similar to Eq. (1) have been derived in nonlinear many-body quantum systems [19] and exciton-polariton condensates [20]. However, in contrast with classical oscillators, detuning can enhance steady-state synchronization of van der Pol oscillators operating in the deep quantum regime [4]. Furthermore, synchronization phase can emerge for identical quantum harmonic oscillators interacting with a common twolevel system [17].

Here, we employ a modified version of the Pearson correlation factor as a measure of synchronization [18] to investigate relationships between synchronization phase, detuning, and 
quantum correlations in open quantum systems exhibiting transient spontaneous synchronization. Our problem of interest is the synchronization of vibrational displacements in a bio-inspired system featuring exciton-vibration interactions, as observed in some photosynthetic complexes (see, for example, Refs. [21-24]). This is an interesting and relevant bio-inspired system because it has been argued that such vibronic interactions are a mechanism for sustaining coherent processes in photosynthetic complexes [23-31]. Thus, investigating this bio-inspired vibronic system can shed light on the synchronization processes and quantum correlations in biophysical systems operating at the interface between the classical and quantum regimes.

We study the dynamics of this bio-inspired system by assuming a detuning between the natural frequencies of the vibrations of interest and show the emergence of a constant phase difference in their spontaneous synchronized states. We discuss how the synchronization phase occurs due to the break of symmetry both in the Hamiltonian and in the dynamics with respect to mode exchange. To gain further insight into the information captured by the synchronization phase, we investigate the quantum correlations between the subsystems of interest as measured by the quantum discord $[32,33]$. We show that, for spontaneous quantum synchronization to emerge, the quantum discord must be greater than classical information at all times. We further show that our synchronization measure is able to capture a change in quantum discord between subsystems as a function of frequency detuning. By extending our analysis to the model considered in Ref. [17], we note that the identified relationships between synchronization, detuning, and quantum correlations hold in a variety of open quantum system scenarios.

This paper is organized as follows: In Sec. II, we describe the bio-inspired vibronic system, its open-system evolution, and the measure for transient spontaneous synchronization we employ. In Sec. III, we present the effects of detuning on synchronization in the dimer. In Sec. IV, we study the relationship between quantum correlations and synchronization phase. In Sec. V, we contrast our results with that of Ref. [17] in which synchronization phase occurs without dependence on detuning. Finally, we conclude in Sec. VI.

Note that, for the rest of the paper, we are focused entirely on transient spontaneous quantum synchronization, thus we will typically refer to it simply as synchronization.

\section{MODELLING TRANSIENT SPONTANEOUS SYNCHRONIZATION IN EXCITON-VIBRATION DIMERS}

In Sec. II A we introduce the Hamiltonian for the excitonvibration dimer model. In Sec. II B we describe the Markovian master equation for the open quantum system dynamics of the exciton-vibration dimer and our numerical methods. In Sec. IIC we describe the measure used to quantify transient spontaneous quantum synchronization and discuss its limitations.

\section{A. The exciton-vibration dimer with detuning}

The exciton-vibration dimer is a prototype light-harvesting unit formed by a pair of chromophores whose local electronic
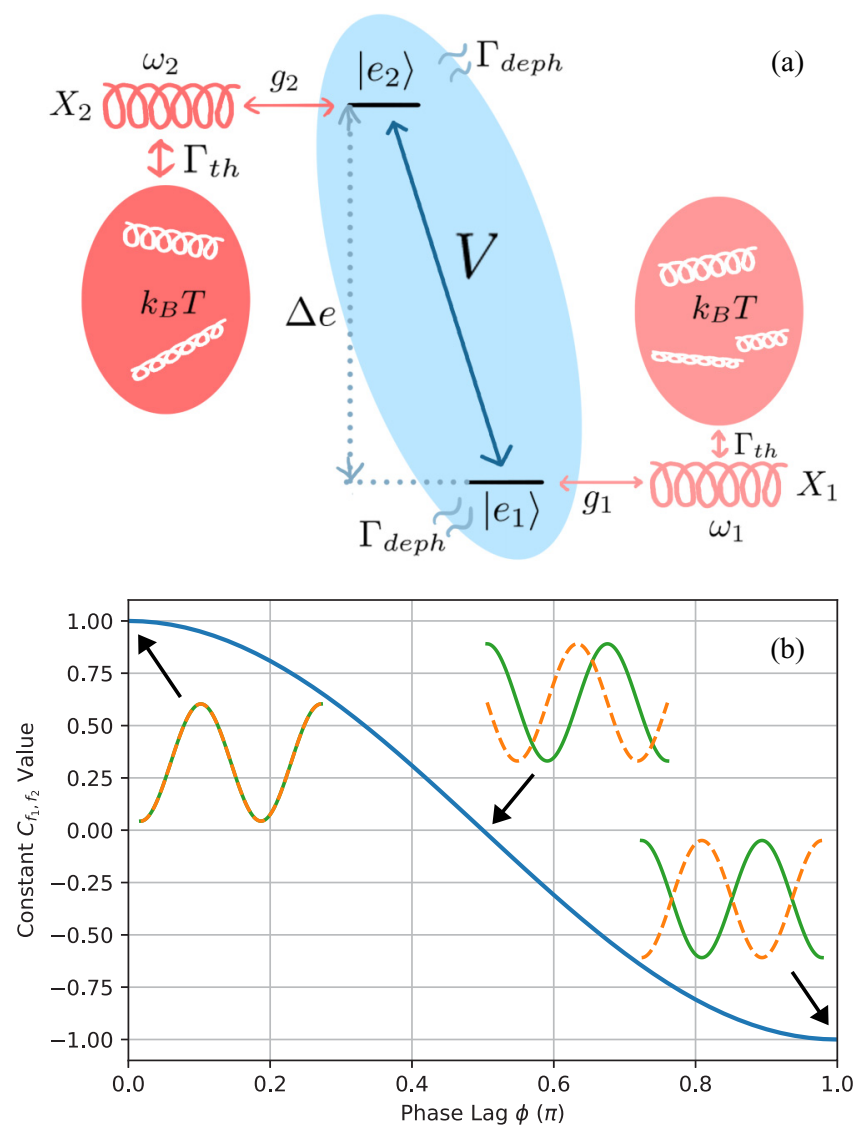

FIG. 1. (a) Schematic diagram of the exciton-vibration dimer. Two chromophores (subscripts $i=1,2$ ) with single excited states $\left|e_{i}\right\rangle$ interact via dipole-dipole coupling of strength $V$. Each electronic state is coupled linearly with strength $g_{i}$ to a harmonic mode of energy $\omega_{i}$. The electronic subsystem (central blue oval) experiences pure dephasing at rate $\Gamma_{\mathrm{deph}}$, and each mode dissipates into separate thermal baths (red ovals) of temperature $k_{B} T$ at rate $\Gamma_{t h}$. (b) Value of the transient spontaneous quantum synchronization measure $C_{f_{1}, f_{2}}(\Delta t=2 \pi / a)=\cos \phi$ [Eq. (18)] for two identical sinusoids $f_{1}, f_{2}$ as a function of their phase difference $\phi$, where $f_{1}=$ $\sin (a t), f_{2}=\sin (a t+\phi)$.

excitations interact with quasicoherent vibrational modes and the system is subjected to relaxation processes due to the surrounding environment [30]. This simplified yet complex enough system is of significant relevance because it captures a main functional unit in natural photosynthetic antennae $[22,25]$. It has been investigated from complementary theoretical perspectives (e.g., see Refs. [26,27,30]) and could be experimentally realized via synthetic methods [34]. We have previously explored synchronization using this model in Ref. [18] and here we introduce a modified version to allow us to explore frequency detuning and synchronization.

The chromophores in the system have single excited states $\left|e_{i=1,2}\right\rangle$ with energies $e_{i=1,2}$, and they interact via dipole-dipole coupling of strength $V$. Each chromophore is then locally coupled to a quantized intramolecular mode of energy $\omega_{i=1,2}$ with strength $g_{i=1,2}$. Figure 1(a) contains a diagram of the dimer model. In this detuned situation, we must account properly for the different reorganization energy contributions to 
the Hamiltonian [35-37] in order to accurately account for the effects of different frequency modes on the dynamics of the exciton-vibration dimer. For clarity, in the following we explicitly derive the dimer Hamiltonian relevant for our situation.

The Hamiltonian for a two-level electronic system, with each site locally and linearly coupled to a vibrational mode $[36,38]$, can be written as follows:

$$
\begin{aligned}
H= & \left(e_{1}+\frac{1}{2} \omega_{1}^{2} d_{1}^{2}\right)\left|e_{1}\right\rangle\left\langle e_{1}\right| \\
& +\left(e_{2}+\frac{1}{2} \omega_{2}^{2} d_{2}^{2}\right)\left|e_{2}\right\rangle\left\langle e_{2}\right| \\
& +\frac{1}{2} \omega_{1}^{2} \hat{x}_{1}^{2}+\frac{1}{2} \hat{p}_{1}^{2}+\frac{1}{2} \omega_{2}^{2} \hat{x}_{2}^{2}+\frac{1}{2} \hat{p}_{2}^{2} \\
& -\omega_{1}^{2} \hat{x}_{1} d_{1}\left|e_{1}\right\rangle\left\langle e_{1}\left|-\omega_{2}^{2} \hat{x}_{2} d_{2}\right| e_{2}\right\rangle\left\langle e_{2}\right| \\
& +V\left(\left|e_{2}\right\rangle\left\langle e_{1}|+| e_{1}\right\rangle\left\langle e_{2}\right|\right),
\end{aligned}
$$

where $e_{i}(i=1,2)$ are the energies of the bare electronic states, $\hat{x}_{i}$ and $\hat{p}_{i}$ are the position and momentum operators of mode $i$ coupled to site $i$, and $d_{i}$ is the displacement of the equilibrium position of mode $i$ due to electronic state $\left|e_{i}\right\rangle$. This displacement is effectively the site-mode coupling strength.

Now we can define the reorganization energy $\lambda_{i}=$ $\frac{1}{2} \omega_{i}^{2} d_{i}^{2}=\omega_{i} S_{i}$ contribution of the mode to the site energy, where $S_{i}$ is the Huang-Rhys factor which is experimentally observable through measurements of the Stokes shift [35]. If $\omega_{1}=\omega_{2}$ and $d_{1}=d_{2}$, the site energies are both shifted by the same amount and the reorganization energy has no effect on dynamics. However, because we are interested in $\omega_{1} \neq \omega_{2}$, the reorganization-energy contributions to site energies cannot be discarded.

We can write the position and momentum operators in terms of the creation and annihilation operators: $\hat{x}_{i}=$ $\frac{1}{\sqrt{2}} \sqrt{\frac{1}{\omega_{i}}}\left(b_{i}^{\dagger}+b_{i}\right)$ and $\hat{p}_{i}=\frac{i}{\sqrt{2}} \sqrt{\omega_{i}}\left(b_{i}^{\dagger}-b_{i}\right)$, where $b_{i=1,2}^{\dagger}$ $\left(b_{i=1,2}\right)$ are the creation (annihilation) operators for the modes. Substituting these into the previous expression (3), we obtain

$$
\begin{aligned}
H= & \left(e_{1}+\omega_{1} S_{1}\right)\left|e_{1}\right\rangle\left\langle e_{1}\left|+\left(e_{2}+\omega_{2} S_{2}\right)\right| e_{2}\right\rangle\left\langle e_{2}\right| \\
& +\omega_{1}\left(b_{1}^{\dagger} b_{1}+\frac{1}{2}\right)+\omega_{2}\left(b_{2}^{\dagger} b_{2}+\frac{1}{2}\right) \\
& -\omega_{1} \sqrt{S_{1}}\left(b_{1}^{\dagger}+b_{1}\right)\left|e_{1}\right\rangle\left\langle e_{1}\right| \\
& -\omega_{2} \sqrt{S_{2}}\left(b_{2}^{\dagger}+b_{2}\right)\left|e_{2}\right\rangle\left\langle e_{2}\right| \\
& +V\left(\left|e_{2}\right\rangle\left\langle e_{1}|+| e_{1}\right\rangle\left\langle e_{2}\right|\right) .
\end{aligned}
$$

We then rotate into a new frequency-dependent exciton basis with matrix $U\left(\tilde{\theta}\left(\omega_{1}, \omega_{2}\right)\right)$ [35],

$$
U=\left(\begin{array}{cc}
\cos \tilde{\theta} & \sin \tilde{\theta} \\
-\sin \tilde{\theta} & \cos \tilde{\theta}
\end{array}\right),
$$

where

$$
\tilde{\theta}\left(\omega_{1}, \omega_{2}\right)=\frac{1}{2} \arctan \left(\frac{2|V|}{\left(e_{2}+\omega_{2} S_{2}\right)-\left(e_{1}+\omega_{1} S_{1}\right)}\right)
$$

is the mixing angle and can be used as a measure of electronic delocalization and hence the exciton size. We then shift the ground-state energy of mode 1 by $\frac{\omega_{1}}{2}$ and mode 2 by $\frac{\omega_{2}}{2}$. The final the total Hamiltonian for our exciton-vibration dimer is then

$$
\begin{aligned}
H= & +E_{1}\left(\omega_{1}, \omega_{2}\right)\left|E_{1}\right\rangle\left\langle E_{1}\left|+E_{2}\left(\omega_{1}, \omega_{2}\right)\right| E_{2}\right\rangle\left\langle E_{2}\right| \\
& +\omega_{1} b_{1}^{\dagger} b_{1}+\omega_{2} b_{2}^{\dagger} b_{2} \\
& +\omega_{1} \sqrt{S_{1}} \tilde{\Theta}_{1} X_{1}+\omega_{2} \sqrt{S_{2}} \tilde{\Theta}_{2} X_{2},
\end{aligned}
$$

where each line corresponds to the exciton Hamiltonian, vibrational Hamiltonian, and exciton-vibration interaction Hamiltonian respectively. We have defined

$$
\tilde{\Theta}=U\left(\tilde{\theta}\left(\omega_{1}, \omega_{2}\right)\right)\left|e_{i}\right\rangle\left\langle e_{i}\right| U^{\dagger}\left(\tilde{\theta}\left(\omega_{1}, \omega_{2}\right)\right),
$$

and the position operator for each mode is $X_{i=1,2}=b_{i}+$ $b_{i}^{\dagger}$. The excitons $\left|E_{d=1,2}\right\rangle$ have eigenenergies that are modefrequency dependent:

$$
\begin{aligned}
E_{i=1,2}\left(\omega_{1}, \omega_{2}\right)= & \frac{1}{2}\left[\left(e_{1}+\omega_{1} S_{1}\right)+\left(e_{2}+\omega_{2} S_{2}\right)\right. \\
& \left.+(-1) \sqrt{\Delta e^{2}\left(\omega_{1}, \omega_{2}\right)+4 V^{2}}\right],
\end{aligned}
$$

where $\Delta e\left(\omega_{1}, \omega_{2}\right)=\left(e_{2}+\omega_{2} S_{2}\right)-\left(e_{1}+\omega_{1} S_{1}\right)$.

The eigenstates of the total Hamiltonian $H$ are excitonvibrational, which we can write in the local basis as

$$
\begin{aligned}
\left|\psi_{j}\right\rangle & =\sum_{d=1,2} \alpha_{d}\left|E_{d}\right\rangle \otimes \sum_{n_{1}=1}^{M} \beta_{n_{1}}\left|n_{1}\right\rangle \otimes \sum_{n_{2}=1}^{M} \gamma_{n_{2}}\left|n_{2}\right\rangle \\
& =\sum_{d, n_{1}, n_{2}} c\left(d, n_{1}, n_{2}\right)\left|E_{d}, n_{1}, n_{2}\right\rangle,
\end{aligned}
$$

where eigenstates $\left|\psi_{j}\right\rangle$ are labeled in ascending energy, and $\left|n_{1}\right\rangle \otimes\left|n_{2}\right\rangle$ where $n$ are the Fock state numbers and the subscripts indicate the mode subspace. To obtain convergent dynamics we account for a maximum occupation $M=8$ in each mode.

Note that, by including the reorganization energy, large detunings will have a significant effect on the coherent dynamics of the dimer. In the following, the detunings and associated reorganization energies considered are small enough for the overall features of the excitation dynamics to be subtle yet they do affect the correlations involved in the synchronization process.

In this paper, we consider the regime of weak electronic coupling where $\Delta E \approx \omega>g>V$ which is characteristic of chromophore pairs present in a variety of light-harvesting proteins $[21,25,26,31,39,40]$. We investigate the synchronization of oscillations in the expectation value of the position operators, $\left\langle X_{i}\right\rangle$, which we refer to as local mode displacements. In the regime we consider, excitons are not fully delocalized and excitonic energies are close to the local energies. The vibronic eigenstates are of a quasilocalized nature and an analysis of their dynamics is therefore useful for understanding the synchronization of local mode displacements [18].

\section{B. Open quantum system model}

Let $\rho(t)$ be the density matrix of the exciton-vibration dimer. In addition to its Hamiltonian from Eq. (6), our exciton-vibration system undergoes relaxation and decoherence caused by local environments. We consider these processes to be Markovian and of Lindblad form,

$$
\dot{\rho}(t)=-i[H, \rho(t)]+D_{\mathrm{deph}}[\rho(t)]+D_{t h}[\rho(t)],
$$


TABLE I. Parameters used for numerical calculations representing the central dimer in the cryptophyte photosynthetic antennae PE545 [26,40]. All units in spectroscopic wave numbers $\mathrm{cm}^{-1}$ except for the rightmost two columns which are specified in the table.

\begin{tabular}{lcccccc}
\hline \hline$\Delta e$ & $V$ & $\omega$ & $g$ & $k_{B} T$ & $\Gamma_{t h}$ & $\Gamma_{\text {deph }}$ \\
\hline 1042 & 92 & 1111 & 267.1 & 207.1 & {$[1 \mathrm{ps}]^{-1}$} & {$[0.1 \mathrm{ps}]^{-1}$} \\
\hline \hline
\end{tabular}

where $D_{\text {deph }}$ and $D_{t h}$ are Lindblad superoperators of the form

$$
D_{v}[\rho]=\Gamma_{v}\left(O_{v} \rho O_{v}^{\dagger}-\frac{1}{2} \rho O_{v}^{\dagger} O_{v}-\frac{1}{2} O_{v}^{\dagger} O_{v} \rho\right),
$$

for various operators $O_{v}$ at rates $\Gamma_{\nu}$.

We assume that each electronic system undergoes local pure dephasing [41,42] with operators $\left|e_{1}\right\rangle\left\langle e_{1}\right|$ and $\left|e_{2}\right\rangle\left\langle e_{2}\right|$ at equal rates of $\Gamma_{\text {deph }}=[0.1 \mathrm{ps}]^{-1}$ such that exciton coherence decays in approximately $0.5 \mathrm{ps}$, as suggested for algal photosynthetic proteins [21].

We assume that each mode undergoes relaxation [42] due to thermal reservoirs at temperature $298 \mathrm{~K}\left(207.1 \mathrm{~cm}^{-1}\right)$. This corresponds to transition Lindblad operators $b_{1}$ and $b_{2}$ at rate $\Gamma_{t h}(1+B)$, and $b_{1}^{\dagger}$ and $b_{2}^{\dagger}$ at rate $\Gamma_{t h} B$. Here $B=\left(e^{\frac{\omega}{k_{B} T}}-1\right)^{-1}$ is the mean number of quanta in a thermally occupied mode of frequency $\omega$ and $\Gamma_{t h}=[1 \mathrm{ps}]^{-1}$ is the rate at which modes equilibrate. Table I summarizes the various parameters.

To numerically simulate the model, we flatten the master equation into an ordinary differential equation

$$
|\dot{\rho}(t)\rangle\rangle=\mathcal{L}|\rho(t)\rangle\rangle,
$$

where $\mathcal{L}$ is the Liouvillian superoperator and $|\rho(t)\rangle\rangle$ are flattened density matrices, which can be solved with standard algorithms.

Our initial state is

$$
\rho(0)=\left|E_{2}\right\rangle\left\langle E_{2}\right| \otimes \rho_{1}^{t h} \otimes \rho_{2}^{t h},
$$

where the electronic system starts in the higher-energy excitonic state $\left|E_{2}\right\rangle$, and both intramolecular modes are initially in thermal equilibrium with their respective baths: $\rho_{i}^{\text {th }}=$ $\sum_{n_{i}} P_{n_{i}}\left|n_{i}\right\rangle\left\langle n_{i}\right|$ and $P_{n_{i}}=\left(1-e^{\frac{-\omega}{k_{B} T}}\right) e^{\frac{-n_{i} \omega}{k_{B} T}}$.

\section{Measuring transient spontaneous synchronization}

Typically, the Pearson correlation factor is used in the quantum synchronization literature because it gives a clear indication of synchronization and shows some information about the timescales involved. For any two time-dependent functions $f_{1}(t)$ and $f_{2}(t)$, the Pearson correlation coefficient is defined as follows [10]:

$$
C_{f_{1}, f_{2}}(t \mid \Delta t)=\frac{\int_{t}^{t+\Delta t} \delta f_{1} \delta f_{2} d t}{\left(\int_{t}^{t+\Delta t} \delta f_{1}^{2} d t \int_{t}^{t+\Delta t} \delta f_{2}^{2} d t\right)^{1 / 2}},
$$

where $\delta f=f-\bar{f}, \bar{f}=\frac{1}{\Delta t} \int_{t}^{t+\Delta t} f\left(t^{\prime}\right) d t^{\prime}$ is a time average, and $\Delta t$ is the averaging window.

However, the usual form of the Pearson correlation factor does not give sufficient information about the early time transient dynamics before synchronization is reached, instead only indicating when it has occurred. Here we present a modification to the Pearson correlation factor that allows it to be used as a continuous measure of phase difference and therefore reveal information about the early times and the emergence of synchronization. This was first presented in Ref. [18]. To do so, we make a particular choice of $\Delta t$, such that our measure of synchronization is

$$
C_{\left\langle X_{1}\right\rangle,\left\langle X_{2}\right\rangle}\left(t \mid \Delta t=\frac{2 \pi}{\omega_{1}}\right),
$$

where $\omega_{1}$ is the frequency of oscillation of the first chromophore, and $\left\langle X_{i}\right\rangle$ are the expectation values of the position operator for each mode. We explain how it works in the remainder of this section.

The Pearson correlation factor returns a value of 1 for positive spontaneous synchronization (in phase), -1 for negative synchronization ( $\pi$ out of phase), and 0 for asynchrony [10]. If we chose $\Delta t$ as close as possible to the time period $T$ of the dominant frequency in $f_{1}$, then the correlation $C_{f_{1}, f_{2}}(t \mid \Delta t=$ $T$ ) becomes a continuous measure of phase difference between the two oscillating signals. This function returns a continuous value in the range of -1 to 1 corresponding to a phase shift of $\pi$ to 0 , respectively, between the functions $f_{1}$ and $f_{2}$.

We derive this relation analytically for two example sinusoids in the following. Consider $f_{1}=\sin \omega t$ and $f_{2}=$ $\sin (\omega t+\phi)$ with identical frequencies and amplitudes that lie within the same range. With the choice of $\Delta t=\frac{2 \pi}{\omega}$, we find that both time averages $\bar{f}_{1}$ and $\bar{f}_{2}$ are zero:

$$
\begin{gathered}
\bar{f}_{1}=\frac{\omega}{2 \pi} \int_{t^{\prime}}^{t^{\prime}+\frac{2 \pi}{\omega}} \sin \omega t d t=0, \\
\bar{f}_{2}=\frac{\omega}{2 \pi} \int_{t^{\prime}}^{t^{\prime}+\frac{2 \pi}{\omega}} \sin (\omega t+\phi) d t=0 .
\end{gathered}
$$

Note that, in general, the functions $f_{1}$ and $f_{2}$ can have different amplitudes or be displaced from zero. Hence, in general, their time averages $\bar{f}_{1}$ and $\bar{f}_{2}$ are not zero. The shifted functions $\delta f_{i}=f_{i}-\bar{f}_{i}$ act to subtract the average value of $f_{i}$ and center any oscillations around zero. This emphasizes the fluctuations around the mean and allows more accurate measurement of phase. For our example $f_{1}$ and $f_{2}$, we have $\delta f_{i}=f_{i}$. The integral of their product is the main measure of synchronization:

$$
\begin{aligned}
\int_{t^{\prime}}^{t^{\prime}+\frac{2 \pi}{\omega}} \delta f_{1} \delta f_{2} d t & =\int_{t^{\prime}}^{t^{\prime}+\frac{2 \pi}{\omega}} \sin (\omega t) \sin (\omega t+\phi) d t \\
& =\frac{\pi \cos \phi}{\omega} .
\end{aligned}
$$

This shows that, for a sliding window of one time period $T=$ $\frac{2 \pi}{\omega}$ and two perfect sinusoids, the time dependence disappears. For any value of $t$, the measure returns a constant value that depends only on the phase difference $\phi$ between the signals.

The denominator in Eq. (13) normalizes the measure to the limits of 1 and -1 . For our example oscillations it takes the value $\left(\int_{t}^{t+\Delta t} \delta f_{1}^{2} d t \int_{t}^{t+\Delta t} \delta f_{2}^{2} d t\right)^{1 / 2}=\frac{\pi}{\omega}$. Together, this finally results in the synchronization measure

$$
C_{f_{1}, f_{2}}(\Delta t=T)=\cos \phi .
$$

In Fig. 1(b), the value of the synchronization function is plotted as a function of constant phase difference $\phi$. 


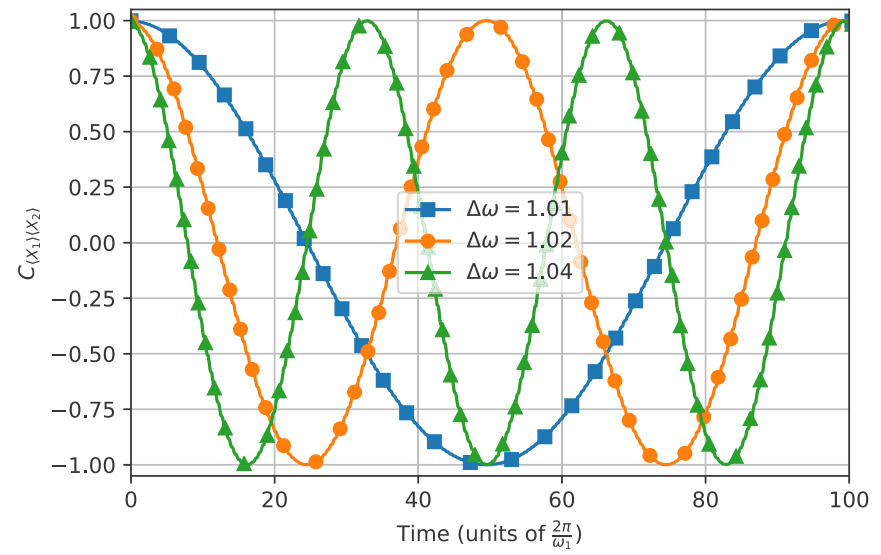

FIG. 2. Time evolution of transient spontaneous synchronization measure $C_{f_{1}, f_{2}}\left(t \mid \Delta t=T_{1}\right)$ for three sets of two uncoupled sinusoids $f_{1}=\sin \left(\omega_{1} t\right), f_{2}=\sin \left(\omega_{2} t+\phi\right)$ with different frequencies $\Delta \omega=$ $\omega_{2} / \omega_{1}$ and constant phase difference $\phi$. Time $t$ given in units of $2 \pi / \omega_{1}$.

The synchronization measure for three different scenarios is illustrated in Fig. 2. We observe that, for two waves of different frequency, the synchronization measure $C_{f_{1}, f_{2}}\left(t \mid \Delta t=T_{1}\right)$ does not stabilize and oscillates as the phase relationship shifts over time. The frequency of oscillation is proportional to the frequency difference between the two oscillators.

These effects can be understood by analyzing the form of Eq. (17). For any two functions, Eq. (17) has a maximum when they are identical, and therefore has a maximum value of 1 . For any phase shift or frequency shift the integral of their product will be less than the square root of the product of their integrals.

This measure is used repeatedly throughout the paper as a dynamic measure of phase and allows us to connect synchronization phase to the Hamiltonian structure and quantum correlations in novel ways. Other attempts to develop a realtime phase measure between two oscillating signals have been conducted along the lines of sliding-window discrete Fourier transform methods [43], Hilbert transforms with data extension [44], and other correlation functions [45].

\section{EMERGENCE OF SYNCHRONIZATION PHASE}

We first consider the effect of detuning in the excitonvibration dimer and find the emergence of a synchronization phase in Sec. III A. Then, we determine the origin of the synchronization phase in Sec. III B, which for our model is due to asymmetric vibronic interactions.

\section{A. Detuning in the bio-inspired dimer}

We define the detuning $\Delta \omega=\omega_{2} / \omega_{1}$ and choose to change the frequency of $\omega_{2}$ only. This allows us to fix the time window of the synchronization measure, $C_{\left\langle X_{1}\right\rangle,\left\langle X_{2}\right\rangle}(t \mid \Delta t)$, as $\Delta t=\frac{2 \pi}{\omega_{1}}$ while still probing the detuning. For simplicity of notation, from here on we denote our time-dependent measure for this time window simply as $C_{\left\langle X_{1}\right\rangle,\left\langle X_{2}\right\rangle}(t)$.

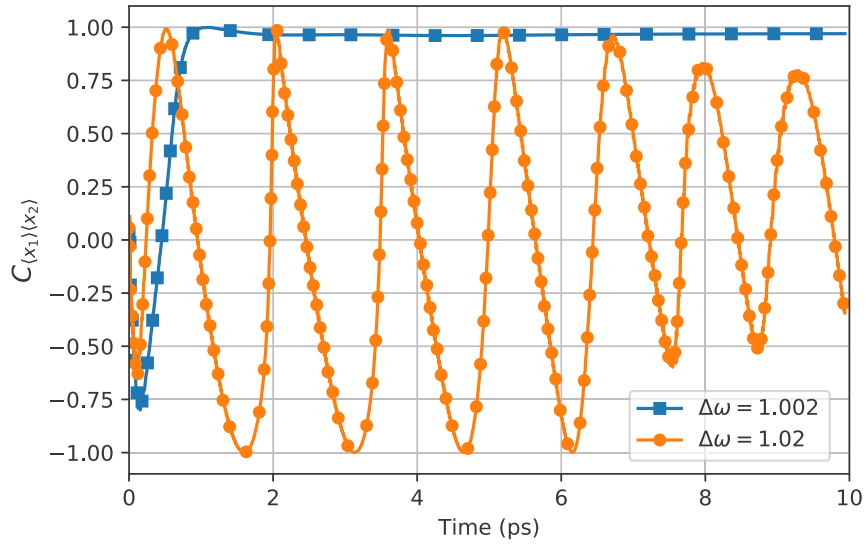

FIG. 3. Dynamics of the transient spontaneous synchronization measure $C_{\left\langle X_{1}\right\rangle,\left\langle X_{2}\right\rangle}(t)$ of the expectation value of mode positions $\left\langle X_{1}\right\rangle,\left\langle X_{2}\right\rangle$ for two regimes of detuning, with frequency difference $\Delta \omega=1.002$ where synchronization occurs, and $\Delta \omega=1.02$ where it does not.

Using the initial state Eq. (12) and the system parameters listed in Table I, we show in Fig. 3 the effects on the synchronization measure for two different regimes of detuning, $\Delta \omega=1.002$ and $\Delta \omega=1.02$. These are chosen to illustrate two distinct scenarios in synchronization dynamics, namely, synchronized and not synchronized, respectively. Recall that here, a constant $C$ value of the measure corresponds to synchronization.

We find that, for detuning $\Delta \omega=1.02$, the synchronization measure oscillates, indicating that the phase between the two oscillators is continuously changing. Hence, the frequencies at which the observables $\left\langle X_{1}\right\rangle$ and $\left\langle X_{2}\right\rangle$ oscillate are different and have not synchronized. We have not shown here, but we also find that the phase oscillation frequencies are correlated with increased detuning. This is as expected from classical dynamics of Eq. (1). The phase relationship $\Delta \phi(t)$ outside the synchronization region would change at a rate proportional to their detuning $d$ with periodic fluctuations from $\epsilon q(\Delta \phi(t))$.

In contrast, we have synchronization in the case of small detuning: the straight line for $\Delta \omega=1.002$ in Fig. 3 indicates that there is a constant, nonzero phase relationship between $\left\langle X_{1}\right\rangle$ and $\left\langle X_{2}\right\rangle$. Their frequencies have synchronized but they are not perfectly aligned in phase. This result also agrees with the predictions of Eq. (1) within the synchronization region.

To take a closer look at this relationship we calculate the synchronization dynamics for a range of detunings, with the results given in Fig. 4. The figure shows only the detunings for which a synchronized state is reached, i.e., having a constant long-time $C_{\left\langle X_{1}\right\rangle,\left\langle X_{2}\right\rangle}$ value. We see a clear negative relationship between the magnitude of detuning and the stable value of $C_{\left\langle X_{1}\right\rangle,\left\langle X_{2}\right\rangle}(t)$ reached, i.e., increasing detuning increases the synchronization phase, up to a breaking point where synchronization can no longer occur.

The overall dynamics of the synchronization measure $C_{\left\langle X_{1}\right\rangle,\left\langle X_{2}\right\rangle}(t)$ at different detunings presented in this section are similar to what would be expected in the classical case. There exists a window of detunings within which synchronization occurs and the time taken to reach a synchronized state increases with detuning. The quantum-mechanical 


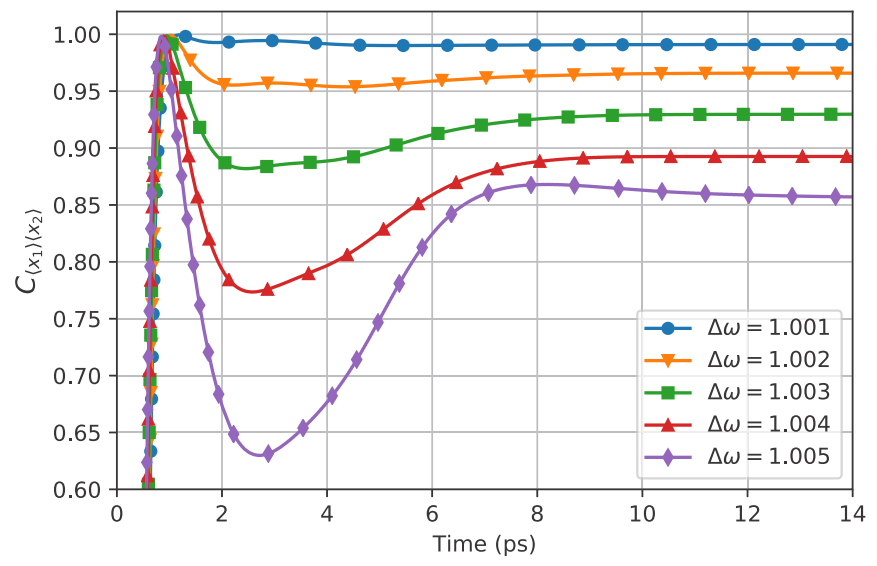

FIG. 4. Dynamics of the transient spontaneous synchronization measure $C_{\left\langle X_{1}\right\rangle,\left\langle X_{2}\right\rangle}(t)$ of the expectation values of mode positions, $\left\langle X_{1}\right\rangle,\left\langle X_{2}\right\rangle$, revealing synchronization phase as a function of detuning, $\Delta \omega$ (listed in the figure). To highlight the long-time behavior, the figure omits the very-short-time regime.

mechanism for this observed behavior is explained fully in the following section.

\section{B. Origin of synchronization phase with detuning}

The origin of the relationship between the synchronization phase and detuning can be understood by considering how the associated changes in the Hamiltonian lead to an asymmetric participation of the local modes in the collective vibronic eigenstates of the system and in the dynamics. The equations of motion for each mode's average displacement are

$$
\begin{aligned}
\left\langle X_{1}\right\rangle & =\sum_{j, k} \rho_{j k}(t) X_{1, k j}, \\
\left\langle X_{2}\right\rangle & =\sum_{j, k} \rho_{j k}(t) X_{2, k j},
\end{aligned}
$$

where $X_{i, k j}=\left\langle\psi_{k}\left|X_{i}\right| \psi_{j}\right\rangle$ with the eigenstates $\left|\psi_{j}\right\rangle$ given in Eq. (8). When $\omega_{1}=\omega_{2}$, the elements $X_{i, k j}$ were restricted to either being equal or opposite upon mode exchange, as thoroughly discussed in Ref. [18]. In this situation each mode participates equally or oppositely in every vibronic coherence (cf. Table 2 in Ref. [18], where the values of $X_{i, k j}$ are equal or scaled by -1 ). Since synchronization is the result of one specific vibronic coherence significantly out-living others, the resultant synchronization phase was restricted to either 0 (when $C_{\left\langle X_{1}\right\rangle,\left\langle X_{2}\right\rangle}=1$ ) or $\pi$ (when $C_{\left\langle X_{1}\right\rangle,\left\langle X_{2}\right\rangle}=-1$ ), depending on which coherence dominated. For the undetuned situation in our bio-inspired system, mode displacements are found to be perfectly synchronized and phase-matched in the long-time regime. Later on in Sec. $\mathrm{V}$ we discuss how this reflects a collective normal mode being effectively decoupled from the electronic dynamics.

With unequal frequencies $\omega_{1} \neq \omega_{2}$, the mode exchange symmetry is broken, leading to an asymmetric participation of each mode both in the vibronic eigenstates and in the dynamics. In the far detuning extreme, one mode may be so far off-resonance with the system energy scales that it does not participate in system dynamics at all and synchronization cannot occur. In the smaller detuning regime, the eigenstates structure do not restrict $X_{1, j k}$ and $X_{2, j k}$ to be symmetric and antisymmetric but instead they have a range of amplitudes. The time-dependent parts of Eq. (19) are identical for $\left\langle X_{1}\right\rangle$ and $\left\langle X_{2}\right\rangle$ but the weights of each oscillating component changes according to $X_{1, j k}$ and $X_{2, j k}$. Within the region of synchronization, these amplitude differences are small and manifest as a constant phase difference between the oscillations of each expected value $\left\langle X_{i}\right\rangle$. This can be seen by expressing each amplitude as $X_{i, k j}=\exp \left(\kappa_{i, k j}\right)$ where $\kappa$ is in general a complex number, thereby contributing a phase to the signal. Outside the region of synchronization, these differences are large, and the signals are composed of different frequencies and have an unstable phase difference.

\section{QUANTUM CORRELATIONS}

Given that the exciton-vibration dimer operates in the quantum regime, a question of interest is how much of its behavior is uniquely quantum? The quantitative relationships between spontaneous quantum synchronization and quantum discord, a measure of purely quantum correlation, have been explored in a range of quantum synchronization settings [13-16,19,46-49]. For example, Ref. [13] finds that the emergence of spontaneous synchronization is correlated with the preservation of quantum discord. Spontaneous synchronization can also be correlated with the generation of entanglement from an initially unentangled state [15], and the quantum mutual information has been proposed as a measure of synchronization that is capable of being used in both deep quantum and semiclassical regimes [46].

In Sec. IV A, we introduce measures of quantum correlations. In Sec. IV B, we investigate the dynamics of quantum correlations between the spontaneously synchronizing subsystems and find further evidence for the connection between synchronization and quantum correlations. Specifically, we reveal that the synchronization phase indicates a change in magnitude of quantum discord between the synchronizing subsystems. This suggests that our adapted synchronization measure can be used to quantify a purely quantum feature.

\section{A. Quantum correlation measures}

The quantum mutual information $I(A: B)$ is a measure of the total correlations between two subsystems $A$ and $B$ of a bipartite quantum system $A B$ and is defined as

$$
I(A: B)=S\left(\rho_{A}\right)+S\left(\rho_{B}\right)-S\left(\rho_{A B}\right),
$$

where $S(\rho)=-\operatorname{tr}[\rho \ln \rho]$ is the von Neumann entropy and density matrices $\rho_{A}=\operatorname{tr}_{B}\left[\rho_{A B}\right], \rho_{B}=\operatorname{tr}_{A}\left[\rho_{A B}\right]$ are subsystems of $\rho_{A B}$. This shared information can be decomposed into classical correlations and quantum correlations. The classical correlations are equivalently the difference in von Neumann entropy of a subsystem before and after a measurement is acted on the other subsystem:

$$
J(B \mid A)=\max _{A_{i}^{\dagger} A_{i}}\left\{S\left(\rho_{B}\right)-\sum_{i} p_{i} S\left(\rho_{B}^{i}\right)\right\},
$$


where

$$
\rho_{B}^{i}=\operatorname{tr}_{A}\left[A_{i}^{\dagger} A_{i} \rho_{A B}\right] / p_{i},
$$

is the residual state of $B$ after measurement of $A_{i}^{\dagger} A_{i}$ (positive operator valued measurements) on subsystem $A$ and $p_{i}=$ $\operatorname{tr}_{A B}\left[A_{i}^{\dagger} A_{i} \rho_{A B}\right]$ is the probability of this outcome. Numerically, the measurements $A_{i}^{\dagger} A_{i}$ are generated randomly until the sum of Eq. (21) satisfactorily converges on its maximum. Note that this equation would be different for the classical correlations from subsystem $A$ to $B$, which we would label $J(A \mid B)$.

The remaining portion of the mutual information that is not classical must be quantum, i.e., the quantum discord $D(B \mid A)$ [32,33]:

$$
D(B \mid A)=I(A: B)-J(B \mid A) .
$$

\section{B. Spontaneous synchronization and quantum correlations}

Given the structure of our dimer model in Sec. II A, the density matrices for the subsystems are accessible. Hence, we can use Eqs. (21), (20), and (23) to calculate the quantum correlations between mode subspaces. Intuitively we would expect some mutual information between modes to be maintained in a synchronized state because, if they were completely uncorrelated, then they should oscillate at independent frequencies and phases. From the work in Ref. [18], we know that, in the systems considered, synchronization requires vibronic eigenstates with sufficient participation from both modes. We postulate that these quantum correlations would not persist in the long-time limit if synchronization is not achieved. The detuning scenarios introduced in this paper provide the ideal regime to test this.

Figure 5 shows numerical calculations of the mutual information, quantum discord and classical information between the two intramolecular modes with PE545 parameters and for two different scenarios: Figure 5(a) considers the case with detuning $\Delta \omega=1.002$ in which synchronization occurs, while Fig. 5(b) considers $\Delta \omega=1.02$ in which synchronization does not occur.

Two time regimes emerge. First, we notice a sharp increase in all correlations from the uncorrelated initial state at $0 \mathrm{ps}$ to a peak at around $0.2 \mathrm{ps}$. Note that the correlations do not originate from the initial state-instead, they must be generated by the coherent interactions in our system. The peak at 0.2 ps coincides with the coherent excitation transfer period that is characteristic of the dynamics in these bio-inspired vibronic dimers [cf. Fig. 8(c) from Ref. [18] and Fig. 10(b) from Ref. [26]]. The excitation transfer mechanism involves transitions between vibronic eigenstates which involve both modes, therefore it is unsurprising that the quantum correlations between the modes also peak at the same time.

Second, we note the decay in correlations from $0.4 \mathrm{ps}$ onward. This behavior is due to the decay of coherent dynamics and the dominance of incoherent processes. In the synchronizing case of Fig. 5(a) we see that the modes remain significantly correlated in the long-time limit whereas in Fig. 5(b) we see that correlations decay rapidly to a much lower value. This clear correlation between synchronization and the preservation of quantum correlations is in agreement with previous findings.
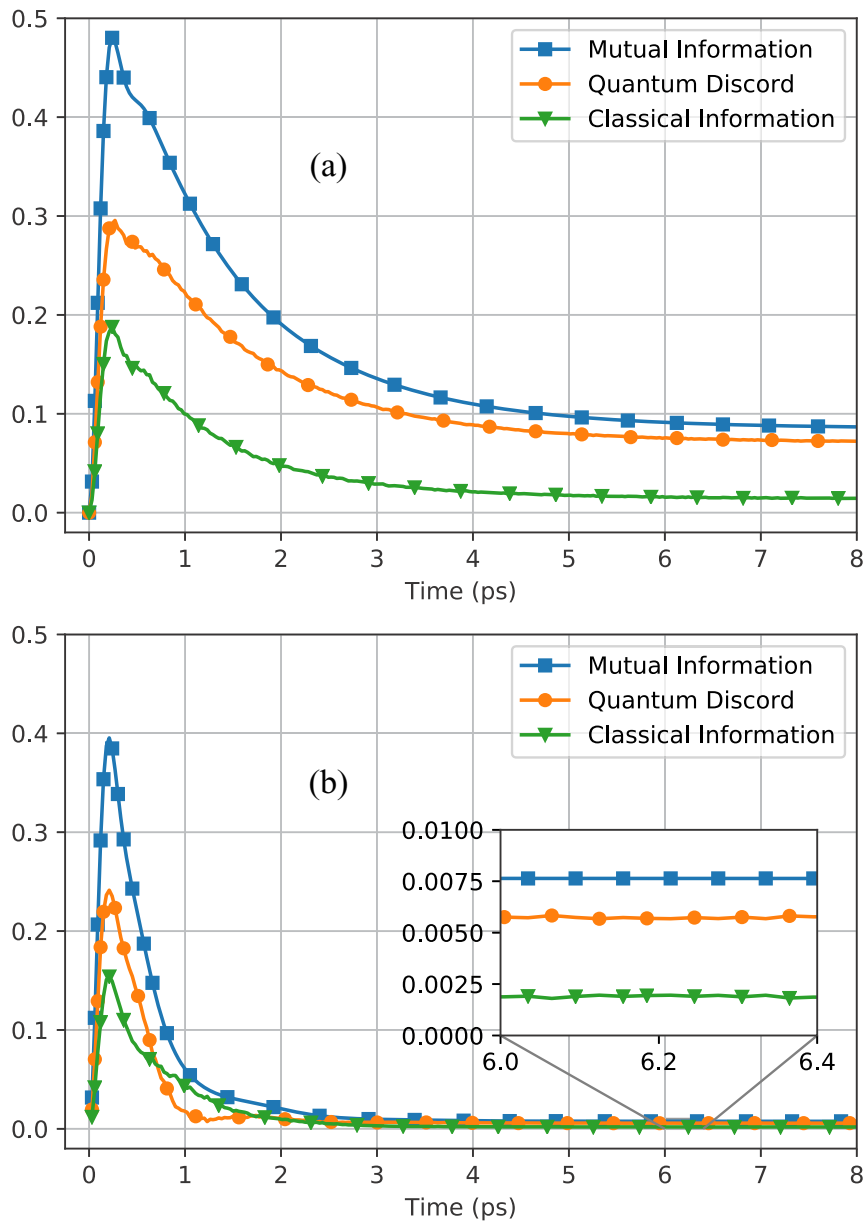

FIG. 5. Dynamics of quantum mutual information, quantum discord, and classical information between mode subsystems in the exciton-vibration dimer model with PE545 parameters (Table I) and initial state (12) for two detuning scenarios: (a) $\Delta \omega=1.002$ for which transient spontaneous synchronization is achieved and (b) $\Delta \omega=1.02$ for which it is not.

Interestingly, we find that, when the systems spontaneously synchronize, the majority fraction of the mutual information consists of quantum discord at every instant in time. In contrast, if the systems do not synchronize, we see a time period in which classical information is greater than quantum discord, as can been noticed in the 1 ps to 2 ps time interval of Fig. 5(b). This leads us to hypothesize a novel dynamical relationship between transient spontaneous synchronization and quantum correlations: for spontaneous quantum synchronization to emerge, the quantum discord must be greater than classical information at all times, whereas for nonsynchronizing cases there may exist time intervals where the greater fraction of correlations are classical.

Now consider the in-between detuning regime for which synchronization phase is also achieved. In Fig. 6, we plot the long-time value of quantum discord (normalized to the discord at no detuning) and compare with the long-time constant synchronization measure $C_{\left\langle X_{1}\right\rangle,\left\langle X_{2}\right\rangle}$. We consider two different exciton delocalization regimes of our dimer model characterized by different values of $\eta=2 V /|\Delta e|$ [18]: (i) the long blue line is for the parameters and detunings from Fig. 4 giving 


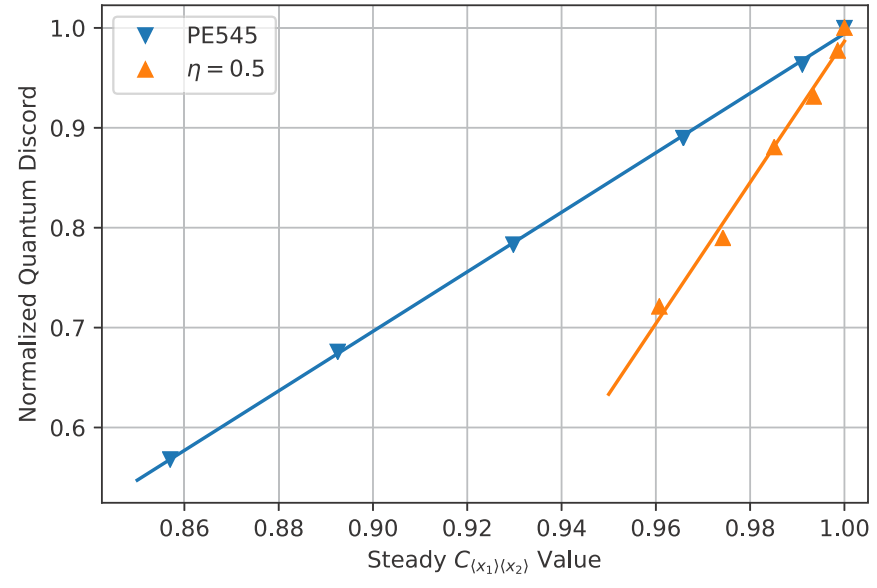

FIG. 6. Long-time stable value of quantum discord between mode subsystems plotted against long-time stable value the synchronization measure $C_{\left\langle X_{1}\right\rangle,\left\langle X_{2}\right\rangle}$. Each data point corresponds to a value of detuning, and quantum discord is normalized to 1 for zero detuning. Two degrees of excitonic delocalization given by $\eta=2 \mathrm{~V} /|\Delta e|$ are considered: (i) $\eta=0.17$ corresponding to parameters as in Table I and (ii) adjusted electronic values such that $\eta=0.5$ but all remaining parameters are the same as in Table I. Linear regressions are plotted by using standard methods to emphasize the relationship.

$\eta=0.17$ and (ii) the short orange line for a slightly more delocalized regime where $\Delta e$ and $V$ are adjusted such that $\eta=0.5$ but all the remaining parameters are as in Table I. Each point corresponds to a different detuning that exhibits a synchronization phase (cf. Sec. III A). Hence, for our bio-inspired system, a larger synchronization phase upon detuning, corresponding to a stable value $0<C_{\left\langle X_{1}\right\rangle,\left\langle X_{2}\right\rangle}<1$, indicates weaker quantum correlations between the modes. This shows that our synchronization measure is able to capture the change in quantum discord between subsystems as a function of frequency detuning. Interestingly, for the quasilocalized regime our system operates, the relationship between the normalized discord

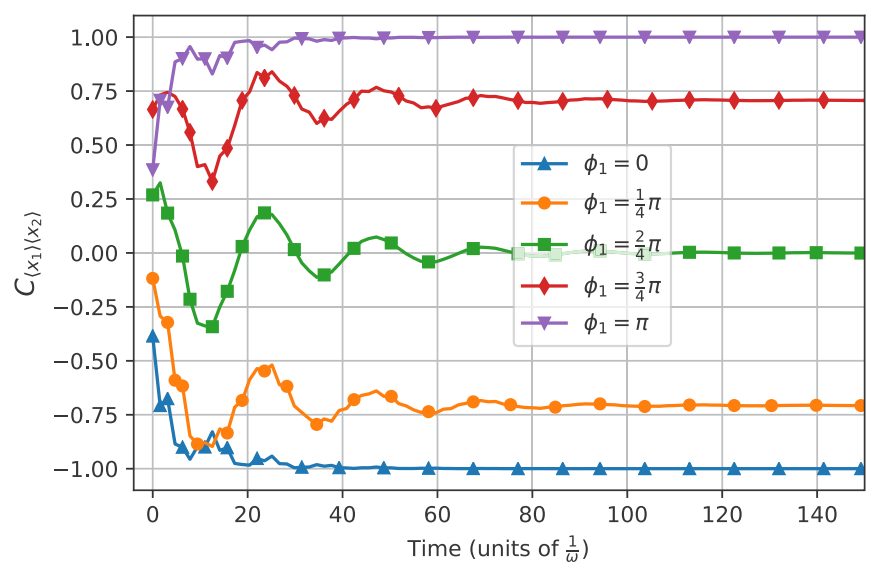

FIG. 7. Dynamics of the transient synchronization measure $C_{\left\langle X_{1}\right\rangle,\left\langle X_{2}\right\rangle}(t)$ of the expectation value of mode positions for different values of (oscillation difference) $\phi_{1}$ as shown in Eq. (27) for the model of Militello et al. [17]. The initial state is $\rho_{0}=$ $\left|e_{1}\right\rangle\left\langle e_{1}\right| \otimes \rho_{\text {vacuum }} \otimes \rho_{\text {coherent }}$. Parameters $\Delta e=e_{2}-e_{1}, \omega=\Delta e, g=$ $\Delta e, \Gamma_{\sigma_{-}}=0.2 \Delta e$. Time $t$ is given in units of $1 / \omega$.

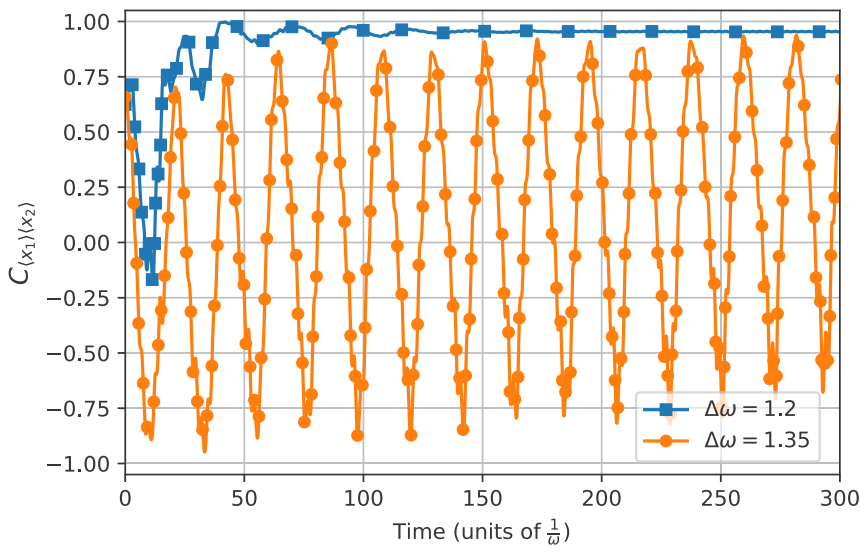

FIG. 8. Dynamics of the transient spontaneous synchronization measure $C_{\left\langle X_{1}\right\rangle,\left\langle X_{2}\right\rangle}(t)$ of the expectation value of mode positions for the model of Militello et al. with $\phi_{1}=\pi$ and $\phi_{2}=0$ and two different detunings: $\Delta \omega=1.2$ where synchronization occurs and $\Delta \omega=$ 1.35 where it does not. The initial state is $\rho_{0}=\left|e_{1}\right\rangle\left\langle e_{1}\right| \otimes \rho_{\text {vacuum }} \otimes$ $\rho_{\text {coherent }}$. Parameters $\Delta e=e_{2}-e_{1}, \omega=\Delta e, g=\Delta e, \Gamma_{\sigma_{-}}=0.2 \Delta e$. Time $t$ is given in units of $1 / \omega$.

and the synchronization phase is linear. The possible reason behind this linearity is that, in this quasilocalized regime, the frequency detuning threshold for which synchronization occurs is much smaller than any relevant system energy scale (see Fig. 4), such that the variations in quantum correlations are small, leading to a relationship with the synchronization phase that appears linear.

In the next section we explore how general our observations are when considering other forms of interaction between two-level systems and Harmonic oscillators. In particular, we show that the trend in which a shift in synchronization phase upon frequency detuning is accompanied by a decrease in quantum correlations holds for a wide range of systems.

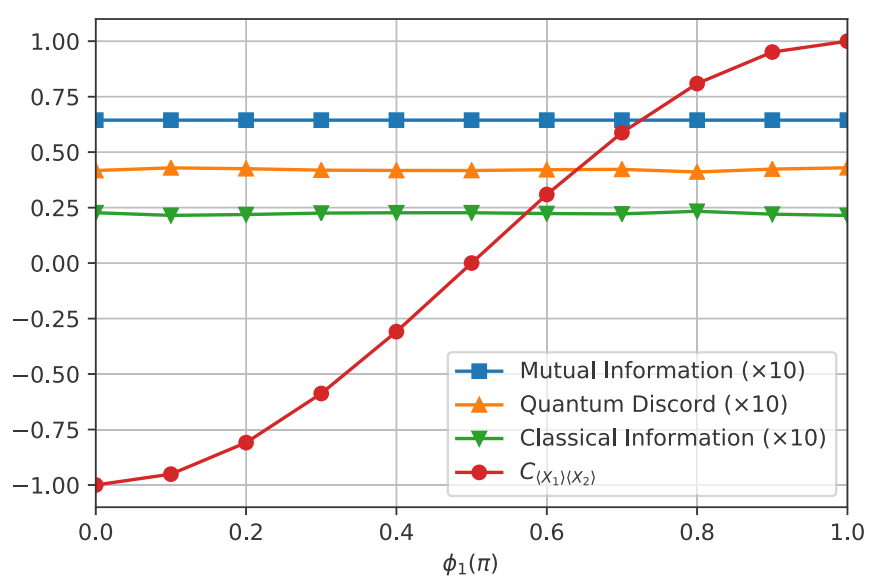

FIG. 9. Long-time-limit stable values of quantum mutual information, quantum discord, and classical information between mode subsystems as functions of $\phi_{1}$ for the interaction Hamiltonian of Militello et al. given in Eq. (24) with $\phi_{2}=0$ and zero detuning. Values for these functions have been scaled by 10 . Also shown is the long-time limit stable value of the synchronization function $C_{\left\langle X_{1}\right\rangle,\left\langle X_{2}\right\rangle}$ as a function of $\phi_{1}$. 

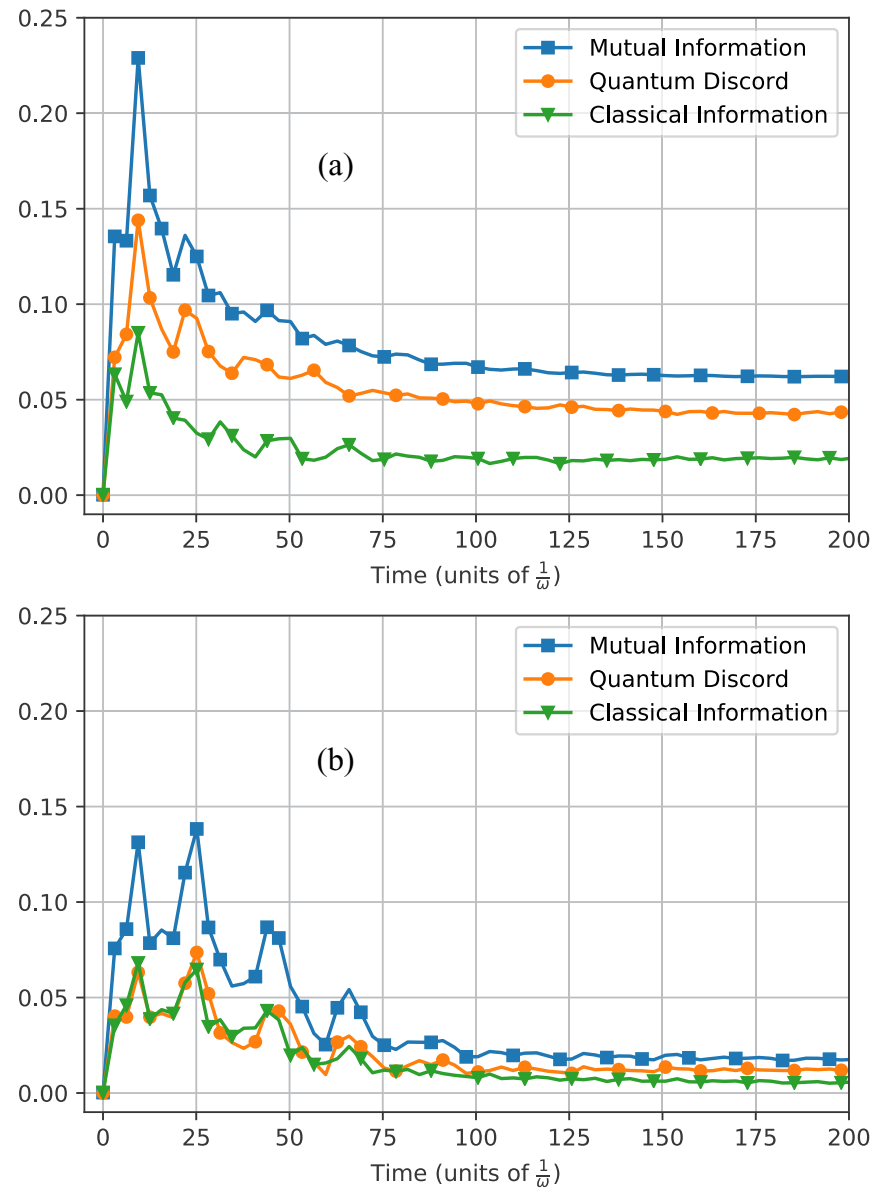

FIG. 10. Dynamics of quantum mutual information, quantum discord, and classical information between mode subsystems in the model of Militello et al. [17] for two detuning scenarios: (a) where synchronization is achieved, $\Delta \omega=1$ and (b) where it is not, $\Delta \omega=$ 1.35. The initial state is $\rho_{0}=\left|e_{1}\right\rangle\left\langle e_{1}\right| \otimes \rho_{\text {vacuum }} \otimes \rho_{\text {coherent }}$. Parameters $\Delta e=e_{2}-e_{1}, \omega=\Delta e, g=\Delta e, \Gamma_{\sigma_{-}}=0.2 \Delta e$. Time $t$ is given in units of $1 / \omega$.

\section{THE CASE OF THE MODEL OF MILITELLO et al.}

To understand how the emergence of the phase synchronization in the presence of detuning is linked to the structure of the Hamiltonian and to the dissipative dynamics the system experiences, we consider the study by Militello et al. [17]. The authors demonstrate synchronization phase in an open quantum system that has comparable features to our bio-inspired model but that exhibits a nonzero synchronization phase in the absence of detuning. We discuss the physical origin of their synchronization phase and demonstrate the agreement with our approach and predictions.

\section{A. Synchronization phase without detuning}

The model considered by Militello et al. consists of a two-level system with ground and excited states denoted as $\left|e_{1}\right\rangle$ and $\left|e_{2}\right\rangle$, respectively, and interacting with two quantum harmonic oscillators whose associated lowering operators are $b_{1}$ and $b_{2}$. The total Hamiltonian has a Jaynes-Cummings form:

$$
\begin{aligned}
H^{\text {Militello }}= & +e_{1}\left|e_{1}\right\rangle\left\langle e_{1}\left|+e_{2}\right| e_{2}\right\rangle\left\langle e_{2}\right| \\
& +\omega_{1} b_{1}^{\dagger} b_{1}+\omega_{2} b_{2}^{\dagger} b_{2} \\
& +g_{1}\left(e^{i \phi_{1}} b_{1}+e^{-i \phi_{1}} b_{1}^{\dagger}\right) \sigma_{x} \\
& +g_{2}\left(e^{i \phi_{2}} b_{2}+e^{-i \phi_{2}} b_{2}^{\dagger}\right) \sigma_{x},
\end{aligned}
$$

where $\sigma_{x}=\left|e_{1}\right\rangle\left\langle e_{2}|+| e_{2}\right\rangle\left\langle e_{1}\right|$ and $\phi_{1}$ and $\phi_{2}$ are phases in the range $0 \leqslant \phi \leqslant \pi$. Each two-level system is also coupled to a zero-temperature reservoir modeled by a Markovian master equation of the form

$$
\dot{\rho}(t)=-i\left[H^{\text {Militello }}, \rho(t)\right]+D_{\sigma_{-}}[\rho(t)],
$$

where $D_{\sigma_{-}}$is a Lindblad superoperator of the form given in Eq. (10) with transition operator $\sigma_{-}=\left|e_{1}\right\rangle\left\langle e_{2}\right|$ and rate $\Gamma_{\sigma_{-}}$. The modes do not experience any direct dissipation.

The authors find that, when synchronization occurs, there is a constant phase difference between the two mode displacements that is determined by the parameters $\phi_{1}$ and $\phi_{2}$ from $H^{\text {Militello }}$ and obeys the relation

$$
\phi_{S}=\pi-\left(\phi_{1}-\phi_{2}\right) .
$$

Note that their approximated synchronization phase has no dependence on detuning and is a function of the variables $\phi_{1}$ and $\phi_{2}$ only. Militello et al. derive this relation by approximating the effects of dissipation on an initial coherent state of the modes. Since the synchronization phase for the model of Militello et al. can emerge for zero-detuning and symmetric coupling strength, i.e., $\omega_{1}=\omega_{2}$ and $g_{1}=g_{2}$, it is important to understand the physical origin such a phase in this situation and how it is subsequently altered as detuning is introduced.

To gain this understanding we focus on the structure of the interaction part of the Hamiltonian in Eq. (24) by fixing $\phi_{2}=$ 0 and letting $\phi_{1}$ control the specific form of the interaction between the modes and the two-level subsystem:

$$
\begin{aligned}
H_{I}^{\text {Militello }}\left(\phi_{1}=0\right) & =g \sigma_{x}\left(X_{2}+X_{1}\right), \\
H_{I}^{\text {Militello }}\left(\phi_{1}=\frac{1}{4} \pi\right) & =g \sigma_{x}\left(X_{2}-\frac{X_{1}}{\sqrt{2}}-\frac{P_{1}}{\sqrt{2}}\right), \\
H_{I}^{\text {Militello }}\left(\phi_{1}=\frac{2}{4} \pi\right) & =g \sigma_{x}\left(X_{2}+P_{1}\right), \\
H_{I}^{\text {Militello }}\left(\phi_{1}=\frac{3}{4} \pi\right) & =g \sigma_{x}\left(X_{2}+\frac{X_{1}}{\sqrt{2}}+\frac{P_{1}}{\sqrt{2}}\right), \\
H_{I}^{\text {Militello }}\left(\phi_{1}=\pi\right) & =g \sigma_{x}\left(X_{2}-X_{1}\right),
\end{aligned}
$$

where $P_{1}$ is the dimensionless momentum operator for mode 1. Evolution in each of these scenarios results in synchronization of the mode observables $\left\langle X_{i}\right\rangle$ with different phase differences $\phi_{S}$. In Fig. 7, we consider the model of Militello et al. and plot the correlation $C_{\left\langle X_{1}\right\rangle,\left\langle X_{2}\right\rangle}(t)$ for the five example interaction Hamiltonians in the set of Eqs. (27). Using the relationship between $C_{\left\langle X_{1}\right\rangle,\left\langle X_{2}\right\rangle}$ and the synchronization phase presented in Fig. 1(b), we can see that our predictions for the synchronization phase agree in all cases with the predictions of Militello et al.

For every case in Eqs. (27) there are effectively two normal collective modes. As dissipation acts directly only on 
the two-level subsystem, the dynamics of the oscillators depend critically on which collective observables are involved

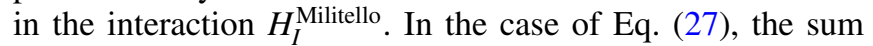
of the mode position operators, also known as the collective "center-of-mass" mode $X_{+}=\left(X_{1}+X_{2}\right)$, is directly coupled to the two-level subsystem. In this case, the collective "relativedisplacement" mode $X_{-}=\left(X_{1}-X_{2}\right)$ is fully decoupled and is therefore free from dissipation. When evolving from an initial coherent state that contains some amplitude in both of these collective modes, the center-of-mass motion decays rapidly while the relative-displacement remains. As we show in Fig. 7, survival of this collective mode in the long-time regime implies perfectly anticorrelated motions $\left(C_{\left\langle X_{1}\right\rangle,\left\langle X_{2}\right\rangle}=\right.$ $-1)$, indicating a constant $\pi$ phase between $\left\langle X_{1}\right\rangle$ and $\left\langle X_{2}\right\rangle$, in agreement with Eq. (26), i.e., $\phi_{S}=\pi-(0-0)=\pi$.

A similar analysis holds for the case of Eq. (27) where the collective center-of-mass motion decouples from the interaction with the two-level system and long-time synchronization of displacements is positive $\left(C_{\left\langle X_{1}\right\rangle,\left\langle X_{2}\right\rangle}=1\right)$ with perfect phase matching, i.e., $\phi_{S}=0$. This case is comparable with our exciton-dimer model with zero-detuning and symmetric coupling, i.e., $\omega_{1}=\omega_{2}$ and $g_{1}=g_{2}=g$, as can be noted by expressing the interaction part of Eq. (6) in terms of effective Pauli matrices for the excitonic system [30]:

$$
H_{I}(\text { dimer })=g\left(\cos 2 \theta \sigma_{z}+\sin 2 \theta \sigma_{x}\right)\left(X_{2}-X_{1}\right),
$$

where $\theta=\frac{1}{2} \arctan (2 V /|\Delta e|)$. For the parameters of our bio-inspired dimer, $\theta$ is small and the interaction with $\sigma_{z}$ dominates, in contrast with $H_{I}^{\text {Militello }}$ where the interaction couples $\sigma_{x}$. The specificity of the $\sigma_{x}$ or $\sigma_{z}$ interaction manifests itself in different short-time synchronization dynamics but the stable long-time synchronization phase is dominated by the effectively decoupled collective mode, as discussed above. We can then conclude that for each in-between value of $\phi_{1}$ and the corresponding Hamiltonians, i.e., Eqs. (27 b)-(27 d), there exists a collective mode that is decoupled both from the two-level system and from the collective mode entering $H_{I}^{\text {Militello }}$, and which dominates the long-time synchronization dynamics, as is indeed discussed by Militello et al. [cf. Eqs. (7) and (8) in Ref. [17] ].

In summary, the synchronization phase predicted by Ref. [17] for zero-detuning is entirely determined by the relative phases of the collective mode that decouples fully both from the two-level system and from the collective mode entering $H_{I}^{\text {Militello }}$. When frequency detuning is introduced, the local mode exchange symmetry is broken, leading to their asymmetric participation in dynamics and to collective modes which cannot be decoupled from each other [cf. Eq. (3a) in Ref. [17] ]. In the presence of a small detuning, synchronization is still determined by a collective mode that decouples from the two-level system but that remains weakly coupled to the collective mode entering the interaction and therefore undergoes indirect dissipation. Thus, a shift in the synchronization phase given by Eq. (26) shall be expected as a function of detuning, as we show in the next section.

\section{B. Detuning in model of Militello et al.}

We now investigate how detuning further influences the synchronization phase in the model of Militelo et al. To understand this we consider the Hamiltonian in Eq. (24) with $\phi_{1}=\pi$ and $\phi_{2}=0$ and detune the mode frequencies such that $\Delta \omega=\omega_{2} / \omega_{1}>1$. The results are reported in Fig. 8 and they strongly resemble the behavior shown in Fig. 3 whereby a small detuning i.e., $\Delta \omega=1.2$ renders a synchronized state with a long-time constant value of $C_{\left\langle X_{1}\right\rangle,\left\langle X_{2}\right\rangle}$ slightly less than 1 , thereby signaling a nonzero phase synchronization. As expected, a larger detuning prevents synchronization.

We expect a similar behavior for all values of $\phi_{1}$ and $\phi_{2}$ when including detuning, with the understanding that the undetuned situation sets the level from which the shift in the synchronization phase should be measured. The results in Fig. 8 also support the understanding that the synchronization phase in the presence of detuning accounts for the asymmetric participation of the modes in the eigenstates and dynamics such that no collective degree of freedom is fully isolated from dissipation. Our analysis then shows that the observations in Sec. III A are not exclusive to the exciton-vibration dimer and apply to a variety of spontaneously synchronizing quantum systems.

\section{Quantum correlations in model of Militello et al.}

In the following we measure the quantum correlations between undetuned modes spontaneously synchronizing with a constant phase as produced by the Hamiltonian of Militello et al. Eq. (24) with identical mode frequencies and interaction strengths.

In Fig. 9 we plot the long-time stable values of quantum mutual information, classical information and quantum discord for a range of $\phi_{1}$ alongside the corresponding long-time stable value $C_{\left\langle X_{1}\right\rangle,\left\langle X_{2}\right\rangle}$ which quantifies the synchronization phase. We find that, under undetuned conditions, the longtime correlations are unchanged as a function of $\phi_{1}$. This is expected since changes in $\phi_{1}$ do not alter the coupling strengths or the individual frequencies of the local modes, and hence shall not affect the long-time correlations. The synchronization value $C_{\left\langle X_{1}\right\rangle,\left\langle X_{2}\right\rangle}$ as a function of $\phi_{1}$ is exactly what we would expect from two sine functions with a constant phase shift between them, as can be seen in the characterization of our synchronization function presented in Fig. 1(b). We do expect that, for a fixed set of $\phi_{1}$ and $\phi_{2}$ parameters, the long-time quantum correlations and synchronization phase will change for different detunings in a similar manner to the results shown in Fig. 6.

Finally, in Fig. 10, we investigate the dynamics of quantum and classical correlations in the model of Militello et al. with $\phi_{1}=\pi$ and $\phi_{2}=0$ and for synchronizing and nonsynchronizing regimes. We find the same qualitative relationship as in the exciton-vibration dimer model with detuning reported in Fig. 5. This supports the generality of the hypothesis introduced in Sec. IV B whereby, for spontaneous synchronization to emerge, the dynamics must be such that quantum discord is larger than the classical information at all times, whereas, in nonsynchronizing systems, classical information can outweigh quantum discord for nonzero time periods. 


\section{CONCLUSION}

In this paper we have defined a synchronization measure that effectively quantifies the synchronization phase between oscillating signals and employed this measure to investigate the dynamics of transient spontaneous synchronization as a function of detuning in a bio-inspired vibronic dimer subjected to Markovian dissipation. We have also explored the quantum and classical nature of the information shared between synchronizing subsystems and how this information dynamics is captured by our proposed synchronization measure.

For the bio-inspired quantum system of interest, where local vibrational modes assist electronic energy transfer, we determined the detuned regime in which spontaneous synchronization can occur with a constant nonzero synchronization phase. Transient spontaneous synchronization in this system reflects the fact that a collective motion undergoes very weak dissipation. In the case of equal oscillator frequencies, the synchronized state in the long-time regime renders zero phase difference between the synchronizing subsystems [18]. We showed that, upon introducing detuning, the synchronization phase is shifted. The origin of this phase shift can be traced back to the asymmetric participation of the vibrations in the joint vibronic eigenstates and in the dynamics. In a normal-mode picture this means that collective normal motions are now coupled to each other such that no collective mode is entirely decoupled from dissipative processes, thereby affecting the phase at which local vibrations synchronize. Through the study of model of Militello et al. [17], we showed that the mechanisms affecting the shift in the synchronization phase under detuned conditions applies to a variety of synchronizing open quantum systems.

We then investigated the relationship between transient spontaneous synchronization and quantum correlations, in both our exciton-vibronic dimer and in the model of Militello et al. [17]. We found that, if synchronization occurs, then the shared information between synchronizing subsystems is primarily quantum discord at all times, whereas in the nonsynchronizing cases classical information may sometimes be the larger fraction. We also found that, as a function of the detuning, the quantum discord between synchronizing subsystems decreases as the synchronization measure decreases; that is, the shift in the synchronization phase upon detuning implies that quantum correlations between the subsystems persist but are diminished. In the case of our bio-inspired system, the relationship between quantum discord and synchronization phase is linear, a fact we attribute to the very small frequency detuning threshold for which synchronization occurs in the quasilocalized regime this system operates. Our results then suggest that our measure is capable of capturing information about a purely quantum property of synchronizing subsystems, and that the measure can indicate the persistent presence of, and change in, quantum discord.

We have considered the simplest bio-inspired quantum scenario, capturing key features present in a variety of natural photosynthetic light-harvesting complexes, to illustrate that quantum synchronization analysis provides an insightful route for understanding truly quantum phenomena in such systems. Thus, our work suggests a promising avenue to investigate nontrivial quantum phenomena in complex biomolecular and chemical systems where molecular vibrations play a fundamental functional role.

\section{ACKNOWLEDGMENTS}

We thank the Engineering and Physical Sciences Research Council (Grant No. EP/L015242/1) and the Gordon and Betty Moore Foundation (Grant No. GBMF8820) for financial support.
[1] A. Pikovsky, M. Rosenblum, and J. Kurths, Synchronization: A Universal Concept in Nonlinear Sciences (Cambridge University Press, Cambridge, 2001).

[2] J. Buck and E. Buck, Nature (London) 211, 562 (1966).

[3] I. Nitsan, S. Drori, Y. E. Lewis, S. Cohen, and S. Tzlil, Nat. Phys. 12, 472 (2016).

[4] N. Lörch, S. E. Nigg, A. Nunnenkamp, R. P. Tiwari, and C. Bruder, Phys. Rev. Lett. 118, 243602 (2017).

[5] G. L. Giorgi, F. Galve, and R. Zambrini, Phys. Rev. A 94, 052121 (2016).

[6] H. Qiu, R. Zambrini, A. Polls, J. Martorell, and B. Juliá-Díaz, Phys. Rev. A 92, 043619 (2015).

[7] M. R. Hush, W. Li, S. Genway, I. Lesanovsky, and A. D. Armour, Phys. Rev. A 91, 061401(R) (2015).

[8] S. Walter, A. Nunnenkamp, and C. Bruder, Phys. Rev. Lett. 112, 094102 (2014).

[9] T. E. Lee and H. R. Sadeghpour, Phys. Rev. Lett. 111, 234101 (2013).

[10] F. Galve, G. Luca Giorgi, and R. Zambrini, Lectures on General Quantum Correlations and their Applications (Springer, Cham, 2017).
[11] H. Eneriz, D. Z. Rossatto, F. A. Cárdenas-López, E. Solano, and M. Sanz, Sci. Rep. 9, 19933 (2019).

[12] A. Cabot, G. L. Giorgi, F. Galve, and R. Zambrini, Phys. Rev. Lett. 123, 023604 (2019).

[13] G. L. Giorgi, F. Galve, G. Manzano, P. Colet, and R. Zambrini, Phys. Rev. A 85, 052101 (2012).

[14] G. L. Giorgi, F. Plastina, G. Francica, and R. Zambrini, Phys. Rev. A 88, 042115 (2013).

[15] C. Benedetti, F. Galve, A. Mandarino, M. G. A. Paris, and R. Zambrini, Phys. Rev. A 94, 052118 (2016).

[16] G. Manzano, F. Galve, G. L. Giorgi, E. Hernández-García, and R. Zambrini, Sci. Rep. 3, 1439 (2013).

[17] B. Militello, H. Nakazato, and A. Napoli, Phys. Rev. A 96, 023862 (2017).

[18] S. Siwiak-Jaszek and A. Olaya-Castro, Faraday Discuss. 216, 38 (2019).

[19] D. Witthaut, S. Wimberger, R. Burioni, and M. Timme, Nat. Commun. 8, 14829 (2017).

[20] M. Wouters, Phys. Rev. B 77, 121302(R) (2008).

[21] G. H. Richards, K. E. Wilk, P. M. G. Curmi, H. M. Quiney, and J. A. Davis, J. Phys. Chem. Lett. 3, 272 (2012). 
[22] J. C. Dean, T. Mirkovic, Z. S. Toa, D. G. Oblinsky, and G. D. Scholes, Chem 1, 858 (2016).

[23] E. Romero, R. Augulis, V. I. Novoderezhkin, M. Ferretti, J. Thieme, D. Zigmantas, and R. van Grondelle, Nat. Phys. 10, 676 (2014).

[24] F. D. Fuller, J. Pan, A. Gelzinis, V. Butkus, S. S. Senlik, D. E. Wilcox, C. F. Yocum, L. Valkunas, D. Abramavicius, and J. P. Ogilvie, Nat. Chem. 6, 706 (2014).

[25] E. Collini, C. Y. Wong, K. E. Wilk, P. M. G. Curmi, P. Brumer, and G. D. Scholes, Nature (London) 463, 644 (2010).

[26] A. Kolli, E. J. O'Reilly, G. D. Scholes, and A. Olaya-Castro, J. Chem. Phys. 137, 174109 (2012).

[27] V. Tiwari, W. K. Peters, and D. M. Jonas, Proc. Natl. Acad. Sci. USA 110, 1203 (2012).

[28] A. W. Chin, J. Prior, R. Rosenbach, F. Caycedo-Soler, S. F. Huelga, and M. B. Plenio, Nat. Phys. 9, 113 (2013).

[29] S. F. Huelga and M. B. Plenio, Contemp. Phys. 54, 181 (2013).

[30] E. J. O'Reilly and A. Olaya-Castro, Nat. Commun. 5, 3012 (2014).

[31] F. Novelli, A. Nazir, G. H. Richards, A. Roozbeh, K. E. Wilk, P. M. G. Curmi, and J. A. Davis, J. Phys. Chem. Lett. 6, 4573 (2015).

[32] L. Henderson and V. Vedral, J. Phys. A: Math. Gen. 34, 6899 (2001).

[33] H. Ollivier and W. H. Zurek, Phys. Rev. Lett. 88, 017901 (2001).

[34] J. Lim, D. Paleček, F. Caycedo-Soler, C. N. Lincoln, J. Prior, H. von Berlepsch, S. F. Huelga, M. B. Plenio, D. Zigmantas, and J. Hauer, Nat. Commun. 6, 7755 (2015).
[35] V. May and O. Kühn, Charge and Energy Transfer Dynamics in Molecular Systems (Wiley-VCH Verlag GmbH \& Co. KGaA, Weinheim, 2011).

[36] T. Renger, Phys. Rev. Lett. 93, 188101 (2004).

[37] R. Stones, Ph.D. thesis, University College London, 2017 (unpublished).

[38] G. C. Schatz and M. A. Ratner, Quantum Mechanics in Chemistry (Dover Publications, New York, 2002).

[39] J. M. Womick and A. M. Moran, J. Phys. Chem. B 115, 1347 (2011).

[40] V. I. Novoderezhkin, A. B. Doust, C. Curutchet, G. D. Scholes, and R. Van Grondelle, Biophys. J. 99, 344 (2010).

[41] H. Haken and G. Strobl, Z. Phys. A: Hadrons Nucl. 262, 135 (1973).

[42] H.-P. Breuer and F. Petruccione, The Theory of Open Quantum Systems (Oxford University Press, Oxford, 2002).

[43] C. M. Orallo, I. Carugati, P. G. Donato, and S. Maestri, Measurement 69, 9 (2015).

[44] Y. L. Shen, Y. Q. Tu, L. J. Chen, and T. A. Shen, Meas. Sci. Technol. 26, 095003 (2015).

[45] Y. L. Shen and Y. Q. Tu, Meas. Sci. Technol. 27, 065006 (2016).

[46] V. Ameri, M. Eghbali-Arani, A. Mari, A. Farace, F. Kheirandish, V. Giovannetti, and R. Fazio, Phys. Rev. A 91, 012301 (2015).

[47] F. Galve, G. L. Giorgi, and R. Zambrini, Phys. Rev. A 81, 062117 (2010).

[48] G. Manzano, F. Galve, and R. Zambrini, Phys. Rev. A 87, 032114 (2013).

[49] B. Zhu, J. Schachenmayer, M. Xu, F. Herrera, J. G. Restrepo, M. J. Holland, and A. M. Rey, New J. Phys. 17, 083063 (2015). 